Supporting Information for

\title{
Imaging Electronic Trap States in Perovskite Thin Films with Combined Fluorescence and Femtosecond Transient Absorption Microscopy
}

\author{
Mary Jane Simpson ${ }^{1}$, Benjamin Doughty ${ }^{1}$, Bin Yang ${ }^{2}$, Kai Xiao ${ }^{2}$, Ying-Zhong Ma*
}

1. Chemical Sciences Division, Oak Ridge National Laboratory, Oak Ridge, Tennessee 37831

2. Center for Nanophase Materials Sciences, Oak Ridge National Laboratory, Oak Ridge,

Tennessee 37831

*Correspondence and requests for materials should be addressed to Y.-Z.M. (email:

may1@ ornl.gov, Phone: 865-574-7213)

This PDF file includes:

Supplementary Methods

Supplementary Figure 1 to 5

Supporting Information References 


\section{Supplementary Methods}

\section{(1) Sample preparation:}

The lead iodide $\left(\mathrm{PbI}_{2}\right)$ solution $(300 \mathrm{mg} / \mathrm{mL}$, dissolved in dimethylformamide) and methylammonium iodide $\left(\mathrm{CH}_{3} \mathrm{NH}_{3} \mathrm{I}\right)$ solution $(40 \mathrm{mg} / \mathrm{mL}$, dissolved in 2-propanol) were sequentially spin-coated onto pre-cleaned glass substrates at $6000 \mathrm{rpm}$ for 30 seconds.

Subsequently, the spun samples were maintained in ambient air for 60 minutes. Finally, the samples were thermally-annealed at $100{ }^{\circ} \mathrm{C}$ for either 60 or 120 minutes in a $\mathrm{N}_{2}$-filled glovebox to form perovskite films. All the samples were covered with $100 \mu$ m-thick microscope glass coverslips and encapsulated with UV epoxy in a $\mathrm{N}_{2}$-filled glovebox. More details about the sample preparation and the morphological characteristics of the prepared films can be found in reference 1 .

\section{(2) Dual-Mode Transient Absorption and Fluorescence Microscope}

Figure S1 shows an illustration of the dual-mode femtosecond transient absorption (TA) and time-integrated photoluminescence (PL) microscope system. 


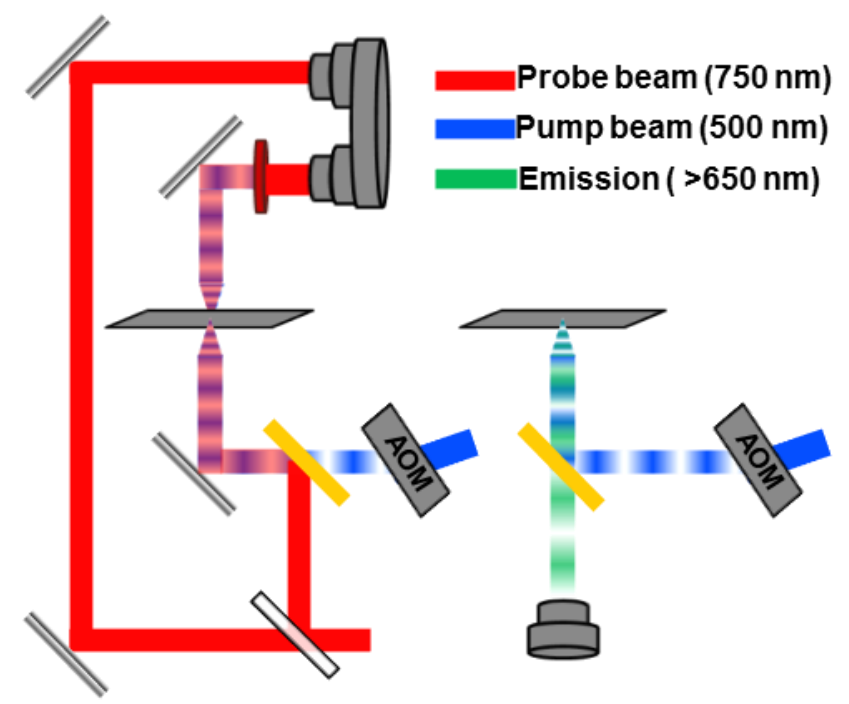

Figure S1: Schematic diagram of the dual-mode femtosecond TA and time-integrated PL microscope. On the left side is the configuration for the TA modality and on the right side is the configuration for the PL modality. To switch between modalities while preserving the region of interest, the dichroic filter and silver mirror are switched with a sliding cube mount. The TA modality utilizes an auto-balanced photoreceiver (Nirvana ${ }^{\mathrm{TM}}$, Newport); the probe beam is split into a signal portion and a reference portion before combining with the pump beam.

\section{Light sources}

The primary light source was a commercial femtosecond Ti:Sapphire regenerative amplifier with a repetition rate of $250 \mathrm{kHz}$, generating $\sim 50 \mathrm{fs}(\mathrm{FWHM})$ pulses centered at 800 $\mathrm{nm}$. The major portion (70 \%) of the amplifier output was used to pump an optical parametric amplifier (OPA) to produce pump pulses centered at $500 \mathrm{~nm}$. A minor portion of the amplifier output $(30 \%)$ was focused onto a sapphire window (10SW8-180, Newport) with 2 mm thickness to generate a single-filament white-light continuum. The remaining fundamental light in the continuum was removed using a $785 \mathrm{~nm}$ short-pass filter (SP01-785RS-25, Semrock), followed by selection of the probe pulse with a $25 \mathrm{~nm}$ (FWHM) bandpass filter centered at $750 \mathrm{~nm}$ 
(86659, Edmund Optics). The probe beam was further attenuated using several absorptive neutral density filters and a beam splitter, with the larger portion serving as a reference for balanced detection. Note that this is a different arrangement from our recently reported femtosecond TAM study, where a probe beam centered at $800 \mathrm{~nm}$ was employed instead. ${ }^{2}$

A dual prism compressor consisting of two SF10 prisms was used to compensate for group velocity dispersion in the pump beam. An acousto-optic modulator (AOM 3200-121, Crystal Technology) was used to intensity modulate the pump beam at $100 \mathrm{kHz}$. The zero order diffracted beam was blocked, and the first order diffraction beam was then temporally delayed using a DC-motor driven optical delay stage with 0.3 fs resolution (UTS150CC, Newport). Finally, the pump beam was combined collinearly with the probe beam using a dichroic mirror (650 SP, Edmund Optics) before beam expansion to fill the back aperture of the microscope objective.

\section{Femtosecond transient absorption microscopy (TAM)}

The pump and probe beams were directed into a modified commercial white light microscope (Eclipse TE 300, Nikon) and focused onto the cover-slipped perovskite samples using a $40 \mathrm{X}, 0.72 \mathrm{NA}$ objective (Nikon). The experimentally measured upper limit of the pump beam spot size (full width at half maximum) is approximately $3.6 \mu \mathrm{m}$ using a razor-edge. Power levels were kept at the lowest possible values while still achieving sufficient signal-to-noise: average power levels were approximately $1 \mu \mathrm{W}$ pump and $5 \mu \mathrm{W}$ probe, corresponding to pulse energies of $5.5 \mathrm{pJ}$ and $12.8 \mathrm{pJ}$, respectively, and pump and probe fluences of $\approx 10 \mu \mathrm{J} / \mathrm{cm}^{2}$ and $\approx$ $6.9 \mu \mathrm{J} / \mathrm{cm}^{2}$, respectively. The transmitted light after the thin film perovskite sample was collected using a $2 \mathrm{X}, 0.06 \mathrm{NA}$ objective and sent through appropriate pump-rejection spectral filters (FGL 
550M, FGL 610 M, Thorlabs) before being focused onto a balanced photodiode (Nirvana Balanced Photodetector, Newport) set to auto-balance with loop bandwidth at 100 .

The transient absorption signal was detected using a lock-in amplifier (SR 810 DSP, Stanford Research Systems), with a typical sensitivity of $100 \mu \mathrm{V}$, a time constant of $10 \mathrm{~ms}$, and a filter with $24 \mathrm{~dB}$ /octave roll off. The lock-in amplifier signal data was read out using a dataacquisition card (DAQ, National Instruments USB-6009). TAM images corresponding to a 128 pixel $\times 128$ pixel data array were collected by scanning the motor-driven microscope $x-y$ stage over a $20 \mu \mathrm{m} \times 20 \mu \mathrm{m}$ area at each chosen delay time between the pump and probe pulses. The lock-in amplifier time constant and specified pixel size determined the $\mathrm{x}-\mathrm{y}$ stage velocity used during image acquisition. Typically, 20-30 TAM images were collected at different delay times, with dense sampling chosen when the transient absorption response changed rapidly and more sparse delay times chosen at longer asymptotic time-regions.

Control of the delay stage, microscope sample scanning $x-y$ stage, lock-in amplifier and DAQ was accomplished using home-written LabVIEW@ programs. Custom data readout software based on LabVIEWC and Python was also written to facilitate image data analysis.

\section{Time-integrated fluorescence microscopy}

For PL microscopy, approximately $70 \mathrm{nW}$ of the intensity-modulated laser pulses centered at $500 \mathrm{~nm}$ was used to excite the thin film perovskite sample in a similar configuration as the transient absorption microscope (Fig. S1), except that the silver mirror immediately before the sample was replaced with a dichroic mirror via a sliding cube mount, which permitted reflection of the excitation beam while also passing the backward propagating fluorescence emission. The excitation power corresponds to a pulse energy of $0.39 \mathrm{pJ}$ and an excitation 
fluence of $\approx 700 \mathrm{~nJ} / \mathrm{cm}^{2}$, assuming a $100 \%$ reflection from the dichroic mirror. The fluorescence emission was detected with a photomultiplier tube (PMT 77348, Oriel Instruments) in a spectrally integrated manner after the excitation beam was rejected using a long-pass dichroic mirror (96105, B-3A, Nikon) and a long-pass filter (LP02-514RE-25, Semrock). The PMT signal was again directed to the same lock-in amplifier with the reference frequency coming from the AOM driver. The lock-in sensitivity was set to $50 \mu \mathrm{V}$, the time constant was $10 \mathrm{~ms}$, and the output filter had $24 \mathrm{~dB} /$ octave roll off. The same area of sample was imaged sequentially with each modality to facilitate data comparison.

\section{Supplementary Figures}
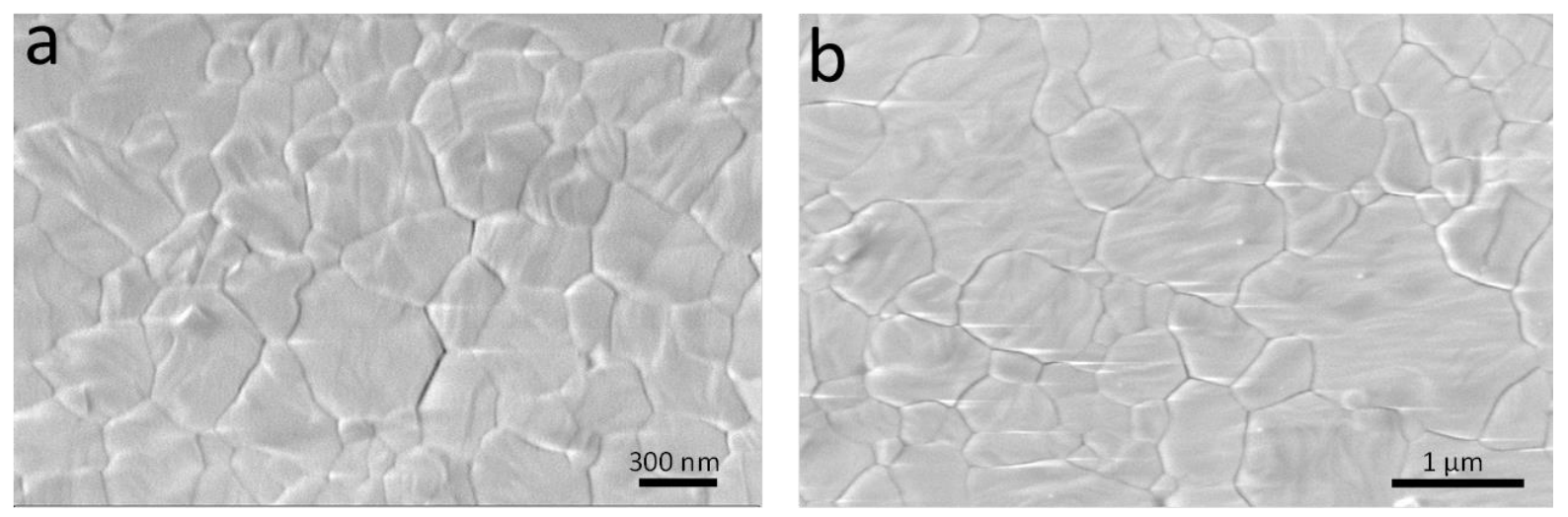

Figure S2: SEM images acquired with Zeiss Merlin Field emission scanning electron microscope under an accelerating voltage of $3 \mathrm{kV}$ for 1 hour (a) and 2 hour (b) annealed perovskite thin films. These thin films were prepared following the same procedure as those used for our optical imaging measurements described in this paper. These images show formation of distinct sizes of single crystal grains, which are about $300 \mathrm{~nm}$ and 1-2 $\mu \mathrm{m}$ for the 1 and 2 hour annealed samples, respectively. 


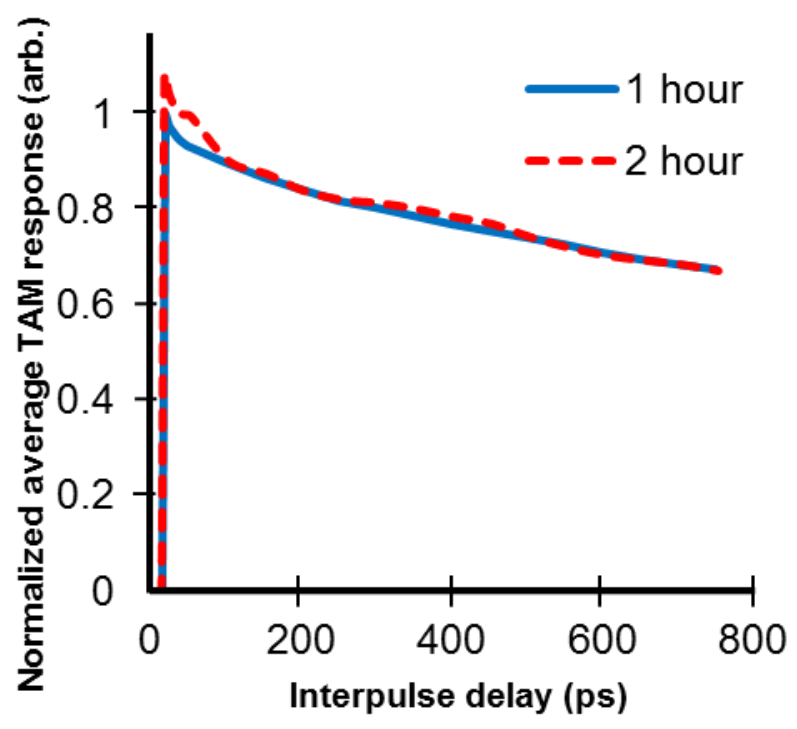

Figure S3: Transient absorption responses obtained by integrating the entire $20 \times 20 \mu \mathrm{m}$ field of view upon normalization at the last data point, which is at $750 \mathrm{ps,} \mathrm{for} 1$ hour (blue solid line) and 2 hour (red dashed line) annealed samples, respectively. 

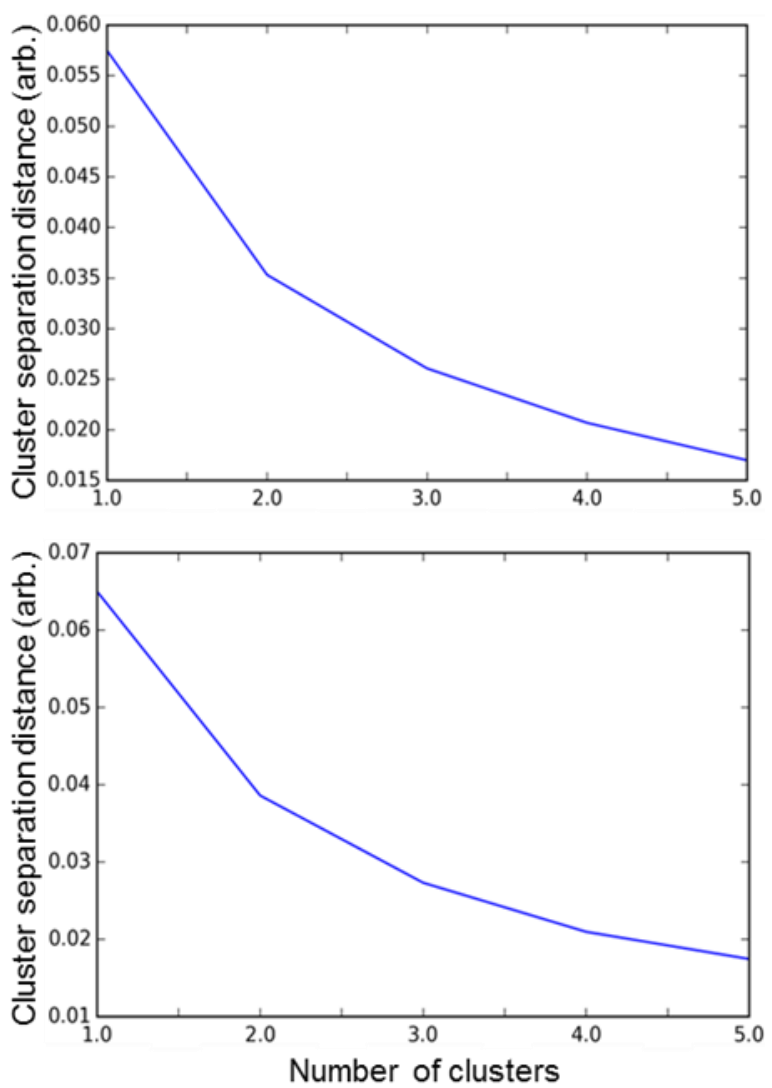

Figure S4: Cluster separation distance plotted as a function of the number of clusters for the 1 hour (top) and 2 hour (bottom) annealed samples. The largest relative improvement to the cluster separation distance occurs when 2 clusters are used. 


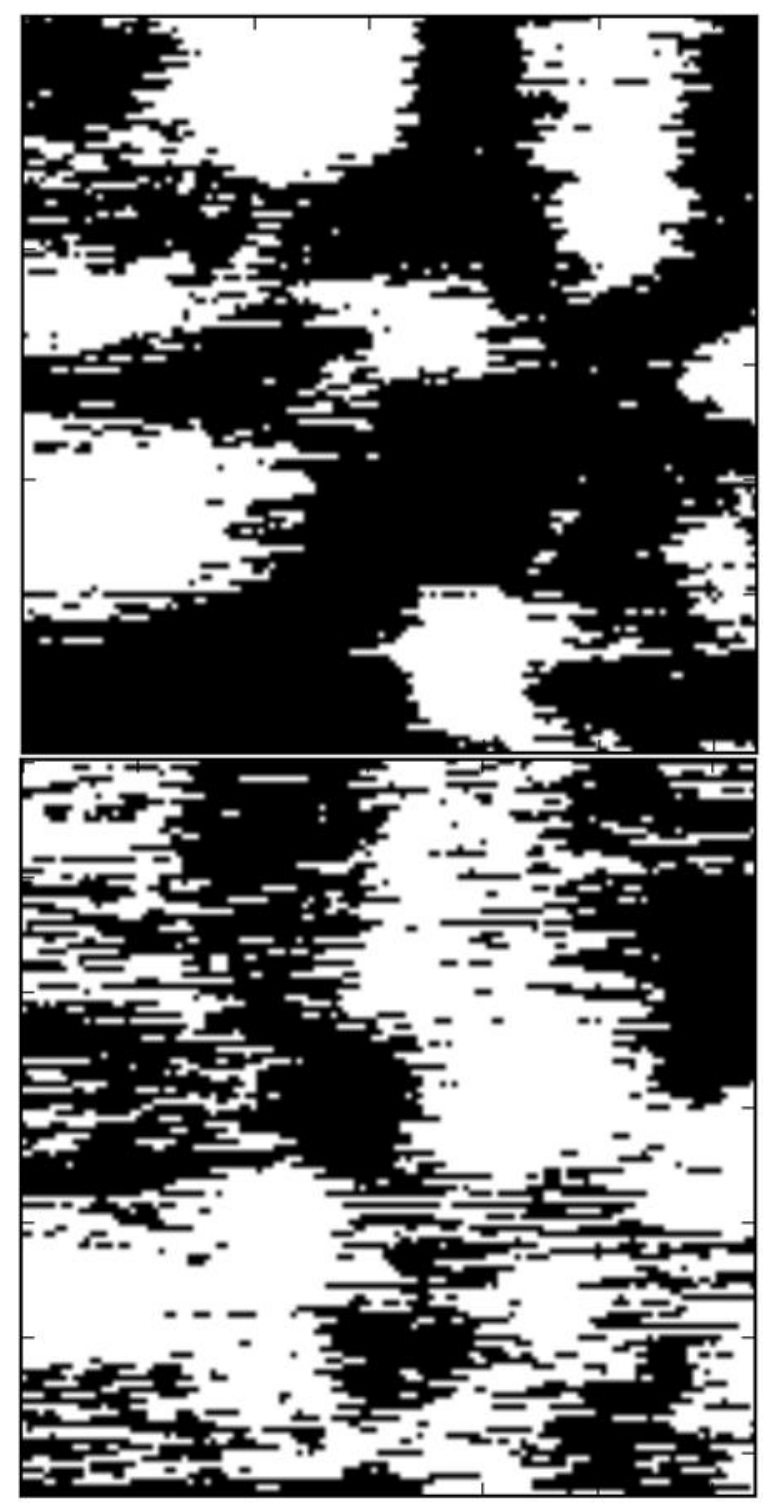

Figure S5: Binary maps of the two clusters found with k-means clustering. Black corresponds to the 'bright' fluorescent regions that do not exhibit losses in PL intensity, and white represents the 'dark' fluorescent regions that do exhibit losses in PL intensity. Images are $20 \times 20 \mu \mathrm{m}$ in size taken at $128 \times 128$ resolution. The 1 hour annealed sample is shown on the top, and the 2 hour annealed sample is shown on the bottom.

\section{Supporting Information References}


1. Yang, B.; Dyck, O.; Poplawsky, J.; Keum, J.; Puretzky, A.; Das, S.; Ivanov, I.; Rouleau, C.; Duscher, G.; Geohegan, D.; Xiao, K. Perovskite Solar Cells with near 100\% Internal Quantum Efficiency Based on Large Single Crystalline Grains and Vertical Bulk Heterojunctions. J. Am. Chem. Soc. 2015, 137, 9210-9213.

2. Simpson, M. J.; Doughty, B.; Yang, B.; Xiao, K.; Ma, Y.-Z. Spatial Localization of Excitons and Charge Carriers in Hybrid Perovskite Thin Films. J. Phys. Chem. Lett. 2015, 6, 3041-3047. 